\title{
Ecological arguments to reconsider data requirements regarding the environmental fate of microbial biocontrol agents in the registration procedure in the European Union
}

\author{
Jürgen Köhl • Kees Booij • Rogier Kolnaar • Willem J. Ravensberg
}

Received: 4 February 2019/Accepted: 13 August 2019/Published online: 19 August 2019

(C) The Author(s) 2019

\begin{abstract}
Microbial biological control agents (MBCAs) against pests and diseases in crops are regarded as sustainable tools in integrated pest management. In the European Union, biological control should be preferred to conventional chemical methods if they provide satisfactory pest reduction. There is no reason to believe that all forms of biocontrol are intrinsically safe. Therefore requirements for registration to assure safety are needed. In the current registration procedure in the European Union, MBCAs are primarily treated as potentially risky organisms that not only produce toxic substances but are also dangerous because they can multiply, spread and genetically adapt. These characteristics give rise to a concern that released MBCAs will spread and become dominant in the environment, resulting in negative effects on other organisms in the natural environment or even humans. These assumption led to
\end{abstract}

Handling Editor: Nicolai Meyling

J. Köhl $(\bowtie) \cdot$ K. Booij

Wageningen University \& Research (WUR),

Wageningen, The Netherlands

e-mail: jurgen.kohl@wur.nl

R. Kolnaar

Linge Agroconsultancy B.V., Oosterhout, The

Netherlands

W. J. Ravensberg

Koppert Biological Systems, Berkel en Rodenrijs, The

Netherlands extensive data requirements that are a time consuming and costly hurdle for bringing MBCAs onto the market. This paper focuses on the relevance and irrelevance of the data requirements for environmental fate and persistence of MBCAs. MBCAs are naturally occurring living organisms that are numerically enhanced to reduce specific plant pathogens or pests. In contrast to chemicals, direct toxicity is not the main mode of action, but rather a variety of mechanisms is involved, including competition, parasitism and activity of secondary metabolites. Their effects and residues cannot be evaluated as done for chemical substances and their breakdown products. Populations of introduced microorganisms always decline due to the natural biological buffering of the environment to levels that are within common fluctuations and ranges without strongly affecting microbial communities. However, the currently used concept of a natural background level as a reference to which densities of MBCAs should decline is of limited value, in particular when endpoints cannot be defined from ecological theory or risk criteria. In conclusion we state that data requirements for persistence could be more freely interpreted in all cases where there is no a priori reason to assume that organisms will not be buffered by the agroecosystem. Since information is only needed 'when relevant', the European Union guidelines leave space for such a proportional interpretation of the data requirements on environmental fate. 
Keywords Biological control - Biological control agents · European Union · Persistence · Populations dynamics $\cdot$ Registration

\section{Regulatory framework for registration of microbial biocontrol agents in the European Union}

Biological control of pests and diseases in crops by microorganisms is generally regarded as a sustainable alternative for conventional chemical plant protection products. Simply by temporally increasing the number of beneficial organisms in a particular area, reduction of unwanted organisms can be achieved. It is assumed that biocontrol has less impact on the environment and human health than conventional chemical control, which is based predominantly on toxicity of single molecules rather than on biological interactions. Through Directive 2009/128/EC to achieve the sustainable use of pesticides, integrated pest management is implemented in the European Union (EU) where sustainable biological, physical and other non-chemical methods must be preferred to chemical methods if they provide satisfactory pest control (Anonymous 2009b).

In the last decades, a wide array of microbial biological control agents (MBCAs) has been developed and proved to be effective against various pests and diseases in a number of crops. New species and strains of microorganisms are continuously discovered and screened for their potential to be applied in biocontrol. Despite some common limitations, many opportunities are foreseen for the use of biocontrol agents (e.g. Bonaterra et al. 2012; van Lenteren et al. 2017).

Even though biocontrol is regarded as more natural than conventional chemical control, the extensive use of MBCAs may nonetheless have an impact on the environment and on human and animal health. There is no reason to believe that all forms of biocontrol are intrinsically safe. Therefor requirements for registration to assure safety are needed, but meanwhile they are also regarded as one of the bottlenecks for new products to be commercialized and applied. In a review of Kiewnick (2007) the development and registration progress of MBCAs has been compared between Northern America and the EU with the conclusion that more extensive data requirements in the EU are a time consuming and costly hurdle for bringing more MBCAs on the market. Also Balog et al. (2017) and Frederiks and Wesseler (2018) compared MBCA registration in different parts of the world and point to regulatory factors in the EU that slow down the progress of MBCA applications in Europe. Safety issues raised in the EU are leading to relatively strict regulations and registration protocols. Before the products can be placed on the market, extensive information is needed about the biological properties of the active organisms.

Originally, the uniform principles and data requirements for registration were derived from those for conventional chemical pesticides, largely neglecting the fact that the properties of microorganisms differ significantly from those of chemicals with regard to specificity, mode of action, and dynamics and behaviour in the environment. The European Food Safety Authority (EFSA) strictly follows the data requirements in the risk assessment which often leads to the identification of data gaps and concerns, e.g. because of missing endpoints, whereas the risk management and approval decision taken by the EU usually is positive. This indicates a fundamental imbalance between the data requirements and the relevance of the data gaps and concerns. Specific registration requirements for MBCAs in the EU were already outlined in 2001 in Directive 2001/36 EC concerning the placing of plant protection products on the market (Anonymous 2001), but instead of simplifying or clarifying procedures, registration requirements were made more complex. The 'chemical' and 'toxicological' approach still reflects in the uniform principles and data requirements for evaluation and approval of plant protection products containing microorganisms in 2011 (Table 1; Anonymous 2011). Precautionary principles and avoiding any theoretical risk predominates the procedures for MBCAs leading to unnecessary and costly data collection. Switching to 'principles of evidence-based acceptable risks' instead would allow more restricted data requirements which may have to be adapted whenever new knowledge and technology becomes available or new safety questions are raised. For example, data on metabolites being formed by a MBCA in situ should not be required, but only when they are present in the formulated product and play an important role in the mode of action. 
Table 1 The uniform principles for evaluation and authorisation of plant protection products and data requirements for active substances concerning survival and persistence of the micro-organism against pests and plant diseases in the European Union

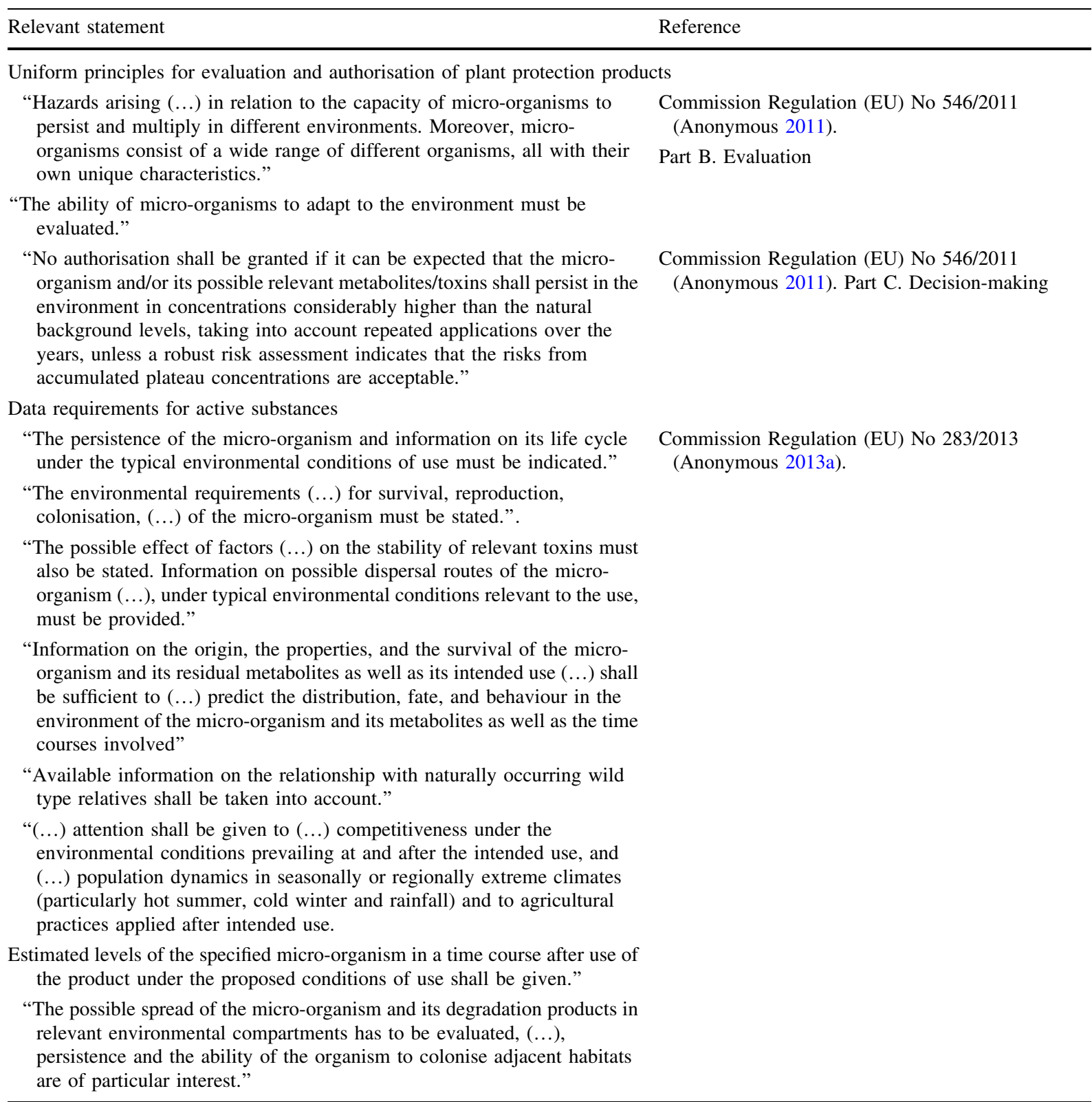

MBCAs were primarily treated as potentially risky organisms that not only produce toxic substances but are also dangerous because they can multiply, spread and genetically adapt. Earlier research reviews (Scheepmaker and Butt 2010) already reported that, in practice, currently applied MBCAs do not pose a risk with regard to proliferation or spread under natural conditions. Also, the risk of toxic secondary metabolites is well-identified and generally manageable for the few MBCAs for which it might be relevant, e.g. for endophytically growing entomopathogenic fungi that potentially can produce metabolites with known toxicity for humans (RiosMoreno et al. 2016).

Despite these scientific discussions and the desire for more biological realism, the data requirements are 
only slightly changed since 2001 . The regulations were adapted in 2009 (Regulation (EC) No 1107/2009; Anonymous 2009a) and Commission Regulation No 546/2011 (Anonymous 2011) replaced the initial uniform principles published in Council Directive 2005/25/EC (Anonymous 2005). However the principles for evaluation and decision making remained the same and the data requirements for evaluation of active substances (Commission Regulation (EU) No 283/2013; Anonymous 2013a) and for plant protection products (Commission Regulation (EU) No 284/2013; Anonymous 2013b) essentially have the same approach, strictly following a precautionary principle. Because of that, the perception of various hazards still dominates the EU methods of risk assessment. Even quite recently, Deising et al. (2017) wrote an opinion paper about unpredictable risks imposed by MBCAs. These fears, however, seem to be far from reality in the practice of biological control (Koch et al. 2018; Lugtenberg 2018). The regulations and guidelines leave space for a proportional interpretation by stating that information is only required when relevant but it is left to the applicants to prove that hazards are irrelevant when this is not obvious for the risk manager. This leads to the need to provide detailed information on all data requirements whether relevant or not which results in undesirable and irrelevant procedural delays in approval (Alabouvette and Cordier 2011). It also leads into inefficient use of resources of both the applicants and the regulators (Frederiks and Wesseler 2018).

For small and medium-sized enterprises involved in the development, production and commercialization of MBCAs, the data requirements for registration are perceived as a road block for further development and innovation. In particular, fulfilling data requirements concerning toxicological issues (e.g. about secondary metabolites) and about environmental fate and persistence are time consuming, costly and, it may be argued, often not justified.

\section{Risk perception of populations of microbial biocontrol agents in the environment}

This paper focuses on the relevance and irrelevance of the data requirements for environmental fate and persistence of MBCAs, as they currently stand in the $\mathrm{EU}$, in order to contribute to a more realistic risk perception for registration requirements and risk assessment. The EU regulation states in Commission Regulation (EU) No 546/2011 (Anonymous 2011) that, for the MBCA to be applied, population background levels and the possibilities for survival, colonisation, reproduction and dispersal must be evaluated: 'Proliferation of indigenous microorganisms should, after a short growth period, level off and continue as for the background microorganisms' (Anonymous 2011). In addition, because microorganisms are able to adapt to the environment, it should be evaluated whether strains selected for biocontrol can survive and multiply better than non-adapted strains. This is based on the idea that 'microbial strains most adapted to the environment can survive and multiply better than the non-adapted strains. Adapted strains have a selective advantage and can form the majority within a population after a number of generations' (Anonymous 2011). These specifications indicate that the regulations are based on a concern or even fear that released MBCAs will spread and become dominant in the environment, resulting in negative effects on other organisms in the natural environment or even humans, since people may be exposed to the organisms themselves or the metabolites they might produce in the food chain. Yet, based on the concerns mentioned above, approval of an MBCA will not be given if the MBCA persists in the environment 'in concentrations considerably higher than the natural background levels, taking into account repeated application over the years, unless a robust risk assessment indicates that the risks from accumulated plateau concentrations are acceptable' (Anonymous 2005, 2011) as discussed in detail by Scheepmaker and Butt (2010).

Ecological knowledge and experiences with regard to release of MBCAs will be discussed in the context of natural dynamics occurring in the environment. Scientific information on microbial ecology and production of secondary metabolites by microorganisms confirm basic microbiological principles which restrict population development in competitive environments with resource limitations and limit the highly regulated production of metabolites to microbial interactions in micro niches (Raaijmakers and Mazzola 2012). With such a background of broadly accepted microbiological knowledge the on-site production of secondary metabolites by MBCAs in the environment can be considered as not relevant for toxicological and ecotoxicological risk assessments (Köhl et al. 2019; 
OECD 2018) which allows a much more efficient registration procedure that focuses on a smaller number of realistic risks. In a similar way, integrating the general principles of microbial population dynamics in the environment will allow to reconsider data requirements on fate and behaviour in the environment. The precautionary principle of the risk assessment can be fulfilled by referring to the general microbiological principles of population dynamics in competitive environments: populations artificially enhanced to higher densities generally decline naturally to levels within their natural density range for the location (Ghoul and Mitri 2016). Enhanced population levels will be subjected to the natural factors that limit and reduce the abundance into the natural range. Detailed population studies for individual MBCAs will thus not be required as long as fitness properties are not changed by genetic modification. Different aspects will be discussed in this paper to develop a risk perception based on ecological arguments:

- How realistic are concerns that microbial biocontrol agents may persist and multiply?

- Natural background levels, dynamics and resilience of microbial ecosystems

- Technologies to monitor released microorganisms

- Proliferation and dispersal

- Examples of populations dynamics of MBCAs after field release

- Synthesis: system dynamics, population densities and risks

Several other review papers have appeared to discuss data requirements for risk assessments of microbials, including discussion of environmental fate issues (Kiewnick 2007; Scheepmaker and Butt 2010; Alabouvette and Cordier 2011; Scheepmaker and van de Kassteele 2011; Mudgal et al. 2013; Sundh and Goettel 2013). This article elaborates on these reviews, with additional information from recent years and with a focus on dynamics and fate of organisms and environments that were not or were only partially covered in earlier reviews.

\section{How realistic are concerns that microbial biocontrol agents may persist and multiply?}

The safety requirements concerning environmental fate and persistence are in line with those for chemical pesticides, however, unlike chemicals, MBCAs are capable of growing, reproducing and potentially spreading. This means that it is difficult to manipulate or to predict their dynamics after release. The public, risk managers and policy makers have concerns when microbial organisms are released in the environment and onto crops for biocontrol because they are uncertain about imaginable risks due to uncontrollable situations. As a result of these uncertainties and possibilities, extensive data on environmental fate, persistence and spread are currently required before new microbial biocontrol agents can be placed on the market. These data are not only difficult, but also costly and time-consuming to attain. Is there sufficient evidence that these data are generally not needed for individual MBCAs if general principles of microbial population dynamics are considered instead?

The question of environmental fate of MBCAs recurs in many publications and policy papers (Kiewnick 2007; Scheepmaker and Butt 2010; Alabouvette and Cordier 2011, Mudgal et al. 2013; Sundh and Goettel 2013; Koch et al. 2018). There is an inherent conflict between the desired control effect, for which temporarily elevated levels are required, and the safety goal that the MBCAs should decline quickly after application.

At first glance, it is understandable that there is concern about what happens in the environment when large amounts of MBCAs are released for biocontrol, and that questions may arise that are analogous with those for chemical pesticides. Data requirements on environmental fate and persistence for registration of MBCAs are primarily based on the assumption that increased populations levels of microbial species can have adverse effects on other organisms and ecosystems (Deising et al. 2017), which is on a non-realistic view on currently used and developed biocontrol agents (Koch et al. 2018; Lugtenberg 2018).

This perception is linked to the image of microbial organisms as pathogenic to humans, animals or plants. Indeed, such pathogenic organisms can become invasive, with fast growing and spreading populations under appropriate circumstances. They are closely associated to hosts that serve as rich food resources. Pathogenic microorganisms may not only cause diseases themselves, but may also produce toxic substances. This means that high densities of such microorganisms can form a risk directly for humans via food and feed products, animals and plants. 
Pathogenic microorganisms do not remain undetected in the environment but are well known and investigated by medical and veterinarian scientists, plant pathologists and ecologists. It is one of the first steps when developing MBCAs to exclude candidates which have been reported as being pathogens of human, vertebrate animals or plants or are closely related to those. Literature databases are used for this check, but also more and more genetic information can be used. An assessment on potential pathogenicity and toxicity is also an essential part of the registration process as regulated by Commission Regulation (EU) No 283/2013 for possible risks on human health and non-target organisms (Anonymous 2013a).

However, though these safety concerns are explainable when it concerns vertebrate pathogens, there are many arguments as to why they are not relevant for MBCAs which have quite different characteristics (Koch et al. 2018). It should be recognized that among the huge numbers of microbial species in the environment, only a few tend to be pathogenic or occur in very high densities. For biological control purposes, MBCAs are selected from the multitude of species that occur naturally in the environment. Current selection procedures (Köhl et al. 2011) focus on antagonistic effectivity and the assurance of nonpathogenic properties. As their origin is mostly from the natural environment, where they occur indigenously and where they are limited in population growth by competitors and resource limitations and do not behave pathogenically, there is no ecological reason to believe that they would do so when released for biocontrol. Formulated MBCAs may contain adjuvants, stickers or specific nutrients to allow a better homogenisation of the spraying suspension during application and a better spread, rain fastness and germination on the target. Formulation additives support the initial establishment of the population, but will not result in long-term protection in the crop from environmental conditions limiting population growth.

Another argument to alleviate concerns about the environmental fate of MBCAs is based on decadeslong experience with applications of non-pathogenic microorganisms having never given rise to uncontrollable situations where the released organisms became dominating species in the habitat (Alabouvette and Cordier 2011; Sundh and Goettel 2013). Deliberate release of microorganisms into the environment to attain effect on ecological processes have been applied for many decades without any apparent problems (Wilson and Lindow 1993). Such releases were not only made to affect undesirable pest and disease species, but also to improve nitrogen fixation, promote growth, for bioremediation, for food and feed processing and as probiotics.

A final argument that should alleviate concerns is that many efficacy trials for biocontrol fail because after application, despite high dose rates, densities tend to decrease rapidly to levels where effectivity is insufficient for control. The main reason for this is that most microorganisms are sensitive to abiotic factors such as draught and UV light, to lack of food resources and to competition with other organisms. Competitive interactions prevail in natural communities (Ghoul and Mitri 2016). As ecological conditions tend to be harsh for most organisms and strains, unnaturally high initial densities tend to decrease rather than increase and persist (Mudgal et al. 2013). Moreover, an artificial rise in density is likely to trigger compensatory ecosystem responses such as density limiting factors, predation and competition. This usually brings the density of the introduced organisms back to within their natural density-range relatively quickly. Consequently, opportunities for explosive growth and spread of microbial populations are assumed to be rare and temporary.

In cases where MBCAs are successful, they have to be applied at high doses, usually above the so-called natural background of the species, to be effective. Maintaining high population levels for some time is a need rather than a concern. Indeed this may conflict with the registration requirements but it does not mean that it is unsafe. It should be realized that even at high application dose or after multiple applications of a MBCA the enhanced population of the applied species is only a minor fraction of the total microbial communities consisting of a magnitude of different species present in a natural system. Typical applications rates of MBCA are $10^{12}$ cells per ha for fungi and $10^{13}$ cells per ha for bacteria. Even a single application of cow manure adds 4000 times more microorganisms to the system than a treatment with a MBCA at $10^{13}$ cells per ha (Lugtenberg 2018). The number of bacteria is $10^{9}$ to $10^{10}$ per gram of fertile agricultural soil (Lugtenberg et al. 2017), which equals to $10^{18}$ to $10^{19}$ bacteria per ha (considering a conservative soil layer of $5 \mathrm{~cm}$ and a soil weight of $1.5 \mathrm{~g} \mathrm{ml}^{-1}$ ). However, near plant roots in the rhizosphere number 
of bacteria per gram soil can even be 10 to 1000 times higher compared to the bulk soil. The number of microorganisms on leaf surfaces is $10^{6}$ to $10^{7}$ per $\mathrm{cm}^{2}$ leaf surface (Koch et al. 2018) which equals to $10^{14}$ to $10^{15}$ microorganisms on leaf surfaces per ha (considering a leaf area index of 1). These examples show that amounts of MBCAs applied for biological control are orders of magnitudes lower compared to the amount of microorganisms already present in the environment or applied via organic fertilizers or other cultivation measures. The impact of other factors such as soil type and tillage, crop types, crop rotation, and cropping practices, mineral fertilizers and pesticide use, use of manure and compost as organic amendments, mulching, irrigation and also climatic influences have tremendous effects on microbial densities (van Veen et al. 1997; Ngosong et al. 2010; Scheepmaker and Butt 2010; Bernard et al. 2012). These examples demonstrate that common agricultural measures have a significant initial impact on microbial communities by adding populations or causing shifts between resident populations. There are no reports of any cases where such initial changes led to unlimited growth of populations of certain species or strains.

Application of MBCAs differs from the examples of adding organic amendments such as manure and composts because single strains are applied instead of complex consortia (including unknown species) and applied amounts of microorganisms are orders of magnitudes lower compared to amounts added through composts or manure. In the history of biocontrol only a few cases have been reported where high artificial densities of released MBCAs could be maintained for longer than a year. This is often welcomed by the end users, as a long lasting protection of crops would make the biocontrol more cost effective and, according to practical experience, without any adverse effects.

Concerns about uncontrolled or even unlimited proliferation and persistence of MPCAs have triggered the environmental fate as a criterion in risk assessment and the requirement that after applications MBCA populations should return to levels not higher that natural background levels. The evidence cited above suggests that concerns about unlimited proliferation are unrealistic for MBCAs.

\section{Natural background levels, dynamics and resilience of microbial ecosystems}

After release, MBCAs are embedded in natural microbial communities that consist of a multitude of species and strains, of which some are highly abundant and many are rare. All species and strains have to compete for scarce nutrients and limited space (Ghoul and Mitri 2016; Yang et al. 2017). Densities of individual species are determined by the availability of resources and substrates that can be competitively colonized. When resources are abundant and conditions favourable, most populations can grow rapidly. Populations are dependent on, and therefore also limited by, the available resources, abiotic conditions and biotic interactions. Both alive or dead organic material as well as water and nutrients can limit the population growth of particular species. Each species has its own set of requirements and tolerance limits that can be narrow or broad.

Interactions between single species in microbiomes can be manifold and complex. In recent microbiome studies technologies have been developed to describe the networks and present a picture of the strength of the interactions and community dynamics (Barberán et al. 2012; Faust and Raes 2012). Such studies also show that competitive interactions between species are abundant and-though communities are dynamic and variable in time-densities of single species fluctuate within restricted ranges due to the interaction with other species. The buffering capacity of microbial communities is accepted as a general and common phenomenon (Lugtenberg 2018; van Elsas et al. 2011; Yang et al. 2017). It is a general principle in ecology that rich biodiversity decrease the susceptibility of an ecosystem to invasion (Tilman 1999). This buffering capacity prevents species from becoming extremely dominant. When the population level of a particular species or strain increases, levels tend then to decline again to lower levels within the "natural range of fluctuating densities" in a given situation. Even though some MBCA strains or species can persist for several months after application, dramatic shifts in microbial communities have not been reported. In contrast, resident communities tend to be not or hardly affected by the application of MBCAs, likely because they are well adapted to the local crop environment and are resilient against introduction of new entomopathogenic microorganisms or antagonists of plant 
pathogens (e.g. Kröber et al. 2014; Mayerhofer et al. 2017, 2019; Perazzoli et al. 2014).

The concept of a natural background density for a species is hard to define within the dynamic and variable nature of microbial communities. Species abundances not only depend on the availability and composition of resources that are variable in time and space, but also on microclimatic conditions and microbial competition. As a result they fluctuate and the existence of a "natural background density" is only a theoretical concept. Even for a specific location or ecological context, densities can be only presented in terms of range and average. When sampling populations for density estimates, even micro-scale variations are relevant, as many organisms are bound to specific substrates and conditions such as the rhizosphere, plant parts, debris, or soil agglomerates. The way MBCAs are applied and thrive at micro-scale levels strongly affects micro-scale densities that are very hard to define or measure. For single strains of a MBCA species as required according to EU regulations, the concept of background density is even more difficult to define, as often such a strain may not be present or detectable in the community before application. This topic has been elaborated by Alabouvette and Cordier (2011), who stress that specific molecular tools are absolutely necessary to monitor the fate of a specific strain. Furthermore, a large and fluctuating fraction of individuals within microbial populations is dormant while only smaller fractions are potentially active or active (Blagodatskaya and Kuzyakov 2013). Background levels based on the total biomass of a population are thus questionable if potential risks arising from the active fraction of the populations are considered.

Of course, within certain system boundaries, e.g. potato field on clay, variation in species densities could be determined to establish a range of densities commonly found in such a system. Whether such a concept would be helpful for defining "background densities" has been explored for entomopathogens used in biocontrol. A methodology to determine natural background levels is suggested by Scheepmaker and Butt (2010) based on samples taken from several areas, soils, crops and seasons. They showed that the natural variation in density of MBCAs is large, making the concept of "background density" disputable.
Apart from specific location and time, population densities of microorganisms can fluctuate strongly due to any biotic or abiotic disturbance in the system. Typical events that change the densities of different species and the composition of microbial communities are husbandry practices (see above). Such interventions thus have significant impact on the size population of a species so that the concept of a "natural background" size of a population is highly questionable.

\section{Technologies to monitor released microorganisms}

Methods to study population dynamics of microbial biocontrol agents have extensively been reviewed by Paulitz (2000). Many different methods are available such as plating, baiting, immunological techniques and DNA based techniques, each with its own advantages and drawbacks. During the last decade, new molecular quantitative approaches with high specificity and sensitivity have been developed and extensively applied. Realtime PCR is broadly used to quantify microbial populations at species or even strain level (Sanzani et al. 2014). Next Generation Sequencing (NGS) techniques are used to characterise microbiome compositions and relative and absolute abundance of microbial groups in the environment (van Elsas and Boersma 2011).

However, monitoring absolute densities in terms of biomass per unit of environmental space is still very challenging. For impact studies, using indirect methods, relative measures and comparing treated and untreated systems can provide useful quantitative information of changes over time. Due to the highly patchy distribution of organisms in different scales, sampling methods and choice of sampling units are crucial aspects of density estimates. Variability in sampling, sample processing and molecular methods can greatly affect the accuracy of the estimates and final conclusions in population studies. As explained in the previous paragraph one should be very cautious to use measurements at different temporal and spatial scales and among systems. In order to make results from modern studies using PCR and other molecular methods comparable with those from those using culturing techniques it is important to note that, for example, DNA-quantities cannot be related to the number of colony forming units (CFU) in a sample. 
Molecular and culturing methods just make different estimates of the abundance of microbial organisms but cannot quantify the exact populations size. Data on populations of MBCAs after application and their natural background are required for risk assessments on the fate in the environment (Anonymous 2013a). A population of a species consists of all individuals of this species living in a defined space. Individual fungal species often produce very different structures: hyphal systems, often consisting of thousands of individual cells, single-cell or multicellular spores, and survival structures, that can be single cell structures such as chlamydospores, or large multicellular structures such as sclerotia, sized up to several centimetres. It is thus impossible to clearly define individuals of such a population consisting of very different structures and to quantify such individuals correctly. There are several methods to estimate the population size of a fungal populations. Plating dilution series on nutrient agar and counting the developed colonies as CFU is a broadly used method. However, many factors have impact on the reliability of CFU counts. CFU counts of a specific species do not only depend on the number of plated single living structures of the particular species in the plated suspension but also on growth medium, incubation temperature, inhibition by in vitro antagonists belonging to other species, or overgrowth by faster growing fungal species. Furthermore, individual colonies have to be identified at species level which practically often is impossible. Fungi forming huge amounts of spores to guarantee population survival (' $r$ strategist') by a relatively small amounts of hyphal cells are significantly overestimated compared to fungi which produce low amounts of spores but are highly resistant to environmental conditions (' $\mathrm{K}$ strategist'). The populations size of a r-strategist will thus be estimated as much higher than of a K-strategist, although the K-strategist may have significantly more impact on the colonized habitat. Consequently, data on possibly biased CFU counts have to be analysed very carefully. A powerful alternative is the quantification of DNA amount of a fungal species (or strain) in a given volume of soil or plant tissue. Realtime PCR methods can be highly reliable in specificity and quantitative in terms of the number of targeted gene sequences. However, also these methods can cause biased data. The efficiency of DNA extraction may differ between substrates and even between fungal structures, e.g. fungal hyphal cells versus resting structures and there is no distinction between DNA from living or dead cells. In cases where it is necessary to follow specific biocontrol strains within a pool of other strains of the same species, a specific molecular identification tool is necessary (Alabouvette and Cordier 2011). The best approach for this is to design a SCAR (specific-characterised-amplifiedregion) marker that can be amplified from samples. This approach was used for example for Trichoderma atroviride (Cordier et al. 2007), Fusarium oxysporum (Edel-Hermann et al. 2011), Pseudomonas brassicacearum (Holmberg et al. 2012) and Bacillus amyloliquefaciens (Gotor-Vila et al. 2016). A further essential step will be the species- or strain-specific quantification of DNA gene sequences using techniques that distinguish between living and dead cells (Daranas et al. 2018). This will allow a realistic quantification of populations derived from released MBCAs in the environment.

\section{Proliferation and dispersal}

For the approval of plant protective MBCAs data are required about the (predicted) distribution, proliferation and spread (dispersal) into the environment (Anonymous 2013a). This is particularly relevant when there are reasons to assume that the distribution extends outside of the local environment where the MBCA will be applied. For many species used as MBCAs it can be assumed that they already occur outside the local site of application even though below application levels. Dispersal outside the area of application may be considered as relevant when increasing densities outside the local situation may cause specific risks.

For control effectivity it may be desirable that the applied MBCA will grow, proliferate, and even disperse on a local scale on root systems and from plant to plant in the system where the target organism is occurring, in order to reach sufficient coverage and suppresses the pest over certain periods of time. A typical example is seen in a MBCA application as seed coating. However, for registration it is often required that information is provided that the organisms does not disperse even without knowing which risk could occur.

The dispersal of an MBCA may occur during application by drift somewhat comparable to 
conventional pesticide drift, although there is a fundamental difference between spraying small particles suspended in water compared to dissolved molecules. Fine droplets susceptible to drift contain dissolved molecules when solutions are applied but cannot contain larger spores as present in suspensions. Dispersal of MBCA also may occur after application by growth (usually at the micro-scale, where bacteria or fungi colonize plants and/or roots systems), by water flow (at the micro-scale, in the soil or by surface water), by splashing and wind (spreading of spores or cells) and by vectors (e.g. the spread of Beauveria by insects).

Dispersal usually has the characteristic of diffusion processes, which means that densities from the source population decline logarithmically and populations are diluted over local environments dependent on the intensity of spread. As long as the MBCA does not establish and reproduce at favourable new spots, densities at a distance from the source are likely to be low or very low. No literature could be found indicating the potential ecological significance of such a diffusive spread. However, it is generally known that pathogenic microorganisms occasionally can have an invasive behaviour when they are non-indigenous and food sources (hosts) are available. Indeed, classical biological control often is based on the principle of establishing and spreading after release. In such cases, data on dynamics and non-target effects are of primary importance. For introduced pathogens of weeds or insect pathogens as control agent this is an issue (van Lenteren et al. 2006). But this is not relevant to MBCAs that are applied against microorganisms if they have no pathogenic life style against animals or plants and in most cases are already present in the region where they are applied.

An obvious distinction in spreading potential can be made between soil and phyllosphere applications. In the soil, MBCAs tend to spread very little and mainly by root colonization, horizontal water movements especially in macropores (Natsch et al. 1996; Yousef et al. 2018), and via husbandry practices such as ploughing. Dispersal in the phyllosphere is more likely by drift during application. Applied and actively produced new spores and cells can be spread by rain droplets and wind. Active spreading structures are formed by applied organisms, such as ballistospores formed by the powdery mildew antagonist Tilletiospis pallescens, which possibly plays an important role in spreading the antagonist from colonized mildew pustules to newly formed pustules in the neighbourhood on the same or on neighbouring leaves. The activity of insect vectors such as soil macrofauna or flying insects may also contribute to dispersal of MBCAs (Meyling et al. 2006 and referred papers).

The process and extent of dispersal, however, has been very little studied over larger scales, i.e. above plant level or in soils. Actively distributing biocontrol agents by means of insects as a vectors such as bees for control of flower pathogens is a challenging field. Dispersal can actively be achieved by using bees or bumble bees as vectors of MBCA from hives with special dispensers which contain the inocula to flowering crops. Such vectoring systems for MBCAs are commercially available. Even combinations of MBCAs against pathogens and against pests are being developed. Combined vectoring of formulated Clonostachys rosea and Beauveria bassiana controlled grey mould and white fly in greenhouse crops (Kapongo et al. 2008). Natural enemies can spread off entomopathogenic fungi. Where it involves MBCAs that pose a potential risk outside the cropping area, the activity range of the insects has to be taken into account. In an elegant study on transmission of a phyllosphere-inhabiting strain of Pseudomonas fuorescens by adult cabbage moths, plant-to-plant transmission could be detected when larvae grew on the cabbage plants inoculated with the $P$. fluorescens strain (Lilley et al. 1997). Of course, longer distance spread from treated plots is imaginable, but this aspect was not further studied. In fact, the spatial spread of the MBCA will be diluted when further away from the source, ultimately to very low concentrations (Lilley et al. 1997).

Information on populations dynamics of MBCAs after application in crops focusses in most cases on relevant targeted parts of the crop, e.g. fruits, leaves or roots, and soil. Spread of applied MBCAs has been monitored after canopy treatments in orchards. Low amounts of applied strains have been found on neighbouring untreated trees (Soto-Muñoz et al. 2015; Vilanova et al. 2018). Transportation of MBCA inocula in soil for few meters by water or animals has also been reported (Troxler et al. 2012). Long distance transportation of MBCAs in air and water may also occur. Concentrations will decrease rapidly with distance from the source, e.g. a field during spray application of a MBCA will add negligible amounts to 
the highly fluctuating natural fungal spore content in the air, often reaching values above $10^{3}$ to $10^{4} \mathrm{~m}^{-3}$ air (Sadyś et al. 2016). Proliferation of MBCAs in air or water is unlikely because they usually are not adapted to these environments. Establishment of minute amounts of MBCAs after drift will follow the same ecological principles as described for MBCA populations after application. There will be a further decline in population size in the competitive environment with limiting resources. It can be concluded that long distance spread across field boarders of MBCAs does not cause relevant risks for air, water or soil.

\section{Examples of population dynamics of MBCAs after field release}

Populations dynamics of MBCAs after field applications have been studied for many MBCAs using a variety of methods from colony counting after plating to strain-specific quantitative real-time PCR. Results give insights into persistence of the MBCA which will strongly depend on environmental conditions. Such information is essential to develop appropriate tactics for the timing of possible re-applications to achieve sufficient control levels. Results are also important for risk assessments on the fate of MBCAs in the environment. Generally, there is a decline of the populations after field applications within days or few weeks. The speed of the decrease depends on the biology of the used antagonist, the targeted habitat, e.g. rhizosphere, soil or crop canopy, and the environmental conditions. The current knowledge on populations dynamics of MBCAs is illustrated, but not reviewed in depth, by examples for academic research on several not yet registered bacterial and fungal MBCAs applied to soil or to canopies for disease or pest control and monitored by strainspecific tools.

\section{MBCAs in soil}

\section{Pseudomonas spp.}

Pseudomonas brassicacearum MA250 is an antagonist of Microdochium nivale causing snow mould in winter cereals (Holmberg et al. 2012). Population studies using strain-specific qPCR were conducted in a field trial with antagonist-coated winter wheat seeds.
The initial population size was $10^{9} \mathrm{CFU}$ per seed. It could be demonstrated that the antagonistic strains colonized the root system of seedlings after one month. However, the estimated cell number of $P$. brassicacearum MA250 per seedling was several orders of magnitudes lower than originally applied with $10^{4}$ to $10^{7}$ cells per seedling. Seven months after seeding, $10^{6}$ to $10^{7}$ cells per seedling were found. During the experiment, low amounts of cells of strain MA250 or closely related strains were also detected in some samples from nearby untreated plots, but it was not possible to definitely conclude on the origin of these signals.

The persistence and spread of strain Pseudomonas protegens $\mathrm{CHAO}$ in soil was investigated in detail with a spontaneous rifampicin mutant (Troxler et al. 2012). The biocontrol strain was released on soil covered or not covered by a ley crop and the number of cells of the strain was determined 72 days after release for different soil habitats. The strain was detected at different soil levels up to $2 \mathrm{~m}$ depth. High populations were found especially in the rhizosphere, earthworm burrows and above plow pan. The main conclusion of the study was that the persistence of the strain fluctuated between micro-habitats. Interestingly, the majority of the cells were viable but non-culturable. The ecological role of such cells which switched to non-culturable stage is still not known. Overall calculations indicated that the release of $10^{13}$ cells resulted in $10^{12}$ cells in different soil habitats after 72 days of which the majority was not culturable.

The population of released $P$. aeruginosa strain GNS.13.2, an endophytic antagonist of Fusarium oxysporum f. sp. cubense in banana, decreased in natural soil within seven days by more than $99 \%$ due to competition with other Pseudomonas strains, even though the released strain initially caused a significant but transient shift in bacterial communities, lasting only one week (Thomas and Sekhar 2016).

Glandorf et al. (2001) introduced Pseudomonas putida WCS358r to soil via treated wheat seeds and quantified the rhizosphere colonization by the antagonist in established wheat crops. In two growing seasons, $10^{7} \mathrm{CFU} \mathrm{g}^{-1}$ rhizosphere samples were detected five days after seeding. Thereafter, the populations decreased down to $10^{2}$ to $10^{3} \mathrm{CFU} \mathrm{g}^{-1}$ at harvest and below detection limit four weeks after harvest. 


\section{Beauveria brongniartii}

Mayerhofer et al. (2015) investigated the long-term persistence of Beauveria brongniartii in soil sampled from 17 sites which had been treated during up to 24 years at high rates of 20 to $40 \mathrm{~kg} \mathrm{ha}^{-1}$ with the biocontrol product Melocont Pilzgerste containing strain B. brongniartii BIPESCO. Samples were plated on selective medium to determine the CFU of Beauveria spp. per gram of soil. Beauveria spp. were detected in samples from eight out of 17 treated sites and also in samples from the three additional sites which had not been treated by the MBCA in the past. Generally, there was a high variation of Beauveria spp. populations within sites. In two sites, both treated with the MBCA during the four years before sampling, Beauveria spp. counts were above the counts of untreated sites. Genetic analysis of the obtained isolates of Beauveria spp. revealed that 27 Beauveria spp. genotypes were detected in samples from various sites, often coexisting with the applied strain $B$. brongniartii BIPESCO. The applied strain was recovered from $41 \%$ of the treated sites, mainly from sites that had been treated last during the 4 years prior to sampling. In one case, the isolate was found in a sample from a site that had been treated for the last time 15 years before sampling. The main conclusions of the long-term study were that repeated applications of $B$. brongniartii BIPESCO are needed to establish sufficient populations and to achieve effective control of the European cockchafer as the targeted pest, and that diverse populations of $B$. brongniartii and other Beauveria spp. consortia can exists on sites repeatedly treated with the specific biological control isolate $B$. brongniartii BIPESCO.

\section{Metarhizium brunneum}

Populations dynamics of the entomopathogenic strain Metarhizium brunneum EAMa 01/58-Su were studied in field trials aiming at the reduction of adult olive fruit fly populations by targeting preimaginals in the soil (Yousef et al. 2017). Soil was inundated beneath the olive tree canopy with the strain at a rate of $1 \times 10^{9}$ conidia per $12 \mathrm{~m}^{2}$ twice per year. No autochthonous strains of Metarhizium spp. were detected in the control plots. After treatments with M. brunneum EAMa $01 / 58-\mathrm{Su}, 0.5$ to $4.5 \times 10^{5} \mathrm{CFU}$ of $M$. brunneum per gram of soil were detected. The concentration in soil dropped rapidly within one month. In one trial, M. brunneum persisted in soil for 12 months with a final concentration of $600 \mathrm{CFU}$ per gram of soil. In two other years, $M$. brunneum EAMa 01/58-Su persisted in soil four months with final concentrations of 80 and $1100 \mathrm{CFU}$ per gram of soil. The short term persistence of the fungus in the soil after inundation treatments was confirmed in additional field observations where M. brunneum CFU counts dropped within four months down to $1600 \mathrm{CFU}$ per gram of soil, the natural background concentration of $M$. brunneum registered in bulk soil (Yousef et al. 2018).

\section{Trichoderma spp.}

The persistence of a Trichoderma virens strain added to soil was studied in a microcosm by Weaver et al. (2005) at three temperature regimes. The initially added population showed an increase during the first days of incubation, then dropped rapidly within the next 50 days and further decreased down during the duration of the experiment of 253 days to the limit of detection limit by plating but never to zero. Similar patterns (but ten-times higher populations sizes) were observed with real-time PCR. The populations dynamics of the mix of a strain of $T$. atroviride and $T$. polysporum added to sterile soil was followed using strain-specific SCAR markers (Feng et al. 2011). Added populations increased during the first days and remained stable or slightly increased during the duration of the experiment of 28 days. However, long-term establishment of the two strains could not be detected in soil of a golf green treated with the MBCAs.

Trichoderma atroviride SC1 applied on soil in vineyards established in the soil, became an integrant part of the local microbial community and could be detected even two years after application (Longa et al. 2009). The strain also spread horizontally on the soil surface several meters during a period of 18 weeks. However, field plots had been inoculated by spreading more than $800 \mathrm{~g}$ of rice kernels grown with $T$. atroviride $\mathrm{SC} 1 \mathrm{~m}^{-2}$ resulting in initial concentrations, e.g. $5 \times 10^{9}$ per gram of soil quantified directly after application in an experiment in 2006, that were several magnitudes higher than expected in commercial applications of MBCAs. Even under these favourable initial conditions, T. atroviride $\mathrm{SC} 1$ declined and 
populations levels were $10^{2}$ to $10^{3}$ per gram of soil after two years, similar to the level of indigenous Trichoderma spp.

The persistence in soil of Penicillium oxalicum strain 212, a MBCA used against Fusarium wilt in tomato and other fungal diseases, was studied in glasshouse and field trials over a time period of 140 days (Vazquez et al. 2013). Using q-PCR techniques it could be shown that populations established well and fluctuated and declined slowly after a couple of months to a level far below the starting concentration at a decline rate depending on soil type. The applications of the specific strain $P$. oxalicum strain 212 did not increase the overall Penicillium populations compared to untreated soil indicating that 'natural background levels' were high. The overall results show that the antagonistic strain persisted in the soil but there was no significant proliferation.

\section{Coniothyrium minitans}

Coniothyrium minitans is an effective mycoparasite against the soil-born plant pathogen Sclerotinia sclerotiorum. The mycoparasite often has been recovered several years after its application. The establishment, multiplication and survival in soils was studied by Bennett et al. (2006) and Yang et al. (2010). In the study of Bennett et al. (2006) it was shown that the long-term survival of the mycoparasite, as a poor competitor for other resources in the soil environment, is associated with the remaining sclerotia of the plant pathogen and that invaded sclerotia are a good reservoir for multiplications and survival of the mycoparasite, where conidia of $C$. minitans are well protected from the environment within pycnidia. This linkage might be responsible for long survival times of the biocontrol agents, but only at low levels and locally in micro-sites in association with its host. The numerical micro-distribution is a headache for sampling. Representative soil sample will show a general decline. Sampling of targeted sclerotia will show a locally and temporarily significant increase in population size. Yang et al. (2010) investigated the survival of $C$. minitans in soil free of its host $S$. sclerotiorum. The antagonist survived at least 750 days although populations dropped from initially $7.2 \times 10^{5}$ to $3.9 \times 10^{4} \mathrm{CFU}$ per gram of soil during that period. Dormancy of conidia may explain the long-term survival also in pathogen-free soil (Yang et al. 2010).
MBCAs in canopies

\section{Pantoea agglomerans}

The fire blight antagonist Pantoea agglomerans E325 was spray-inoculated in apple orchards (BraunKiewnick et al. 2011). On leaves, the antagonistic population was low after application, increased to $10^{3}$ to $10^{4} \mathrm{CFU} \mathrm{g}^{-1}$ after five days and thereafter decreased within two weeks below detection level. On flowers, the antagonist increased initially to $10^{4}$ to $10^{5} \mathrm{CFU}$ per flower and declined during 120 days until harvest. On fruits, $10^{3} \mathrm{CFU}$ per gram of fruit were detected on sepals, but could not be detected on fruit surface.

The environmental fate and behaviour of another strain, Pantoea agglomerans CPA-2, was studied in citrus fruit (Soto-Muñoz et al. 2015). Directly after release in the field, approximately $3.1 \times 10^{4} \mathrm{CFU}$ of the strain $\mathrm{cm}^{-2}$ were found on fruits by plating. There was a drop in populations down to $70 \mathrm{CFU}$ within three days and down to $32 \mathrm{CFU}$ within 16 days. Similar pattern were found using strain-specific qPCR and PMA-qPCR for quantification of viable cells. The overall results on population dynamics of $P$. agglomerans CPA-2 in different habitats in the orchard demonstrate that the strain can persist at low levels but not proliferate on surfaces of fruit and leaves of citrus as well as on weeds. It also did not proliferate in soil.

\section{Pseudomonas fluorescens}

The population dynamics of Pseudomonas fluorescens EPS62e, antagonistic to Erwinia amylovora (fire blight) was monitored on leaves of greenhouse grown pear (Pujol et al. 2005). The initial population of $10^{7}$ per gram of leaf dropped to $10^{4}$ to $10^{5}$ per gram of leaf during 17 days and remained stable until the end of the experiment 28 days after inoculation.

\section{Bacillus amyloliquefaciens}

Strain Bacillus amyloliquefaciens CPA-8, originating from nectarine surface, has been developed for biological control of pre- and post-harvest brown rot caused by Monilinia spp. The population of $B$. amyloliquefaciens CPA-8 was monitored after orchard application using a combination of plating on agar and confirming strain identity by using a strain-specific 
PCR (Vilanova et al. 2018). Spraying bacterial suspensions containing $10^{7}$ cells $\mathrm{ml}^{-1}$ at 31 per tree resulted in $2.7 \times 10^{4} \mathrm{CFU}$ per $\mathrm{cm}^{2}$ of leaf surface of peaches. This value remained stable for 21 days and

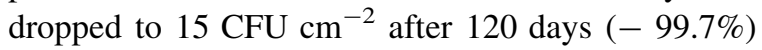
and remained low until the end of the experiment after 180 days. On peach fruit $1.3 \times 10^{4} \mathrm{CFU}$ per $\mathrm{cm}^{2}$ of fruit surface were detected after application. Fruits were harvested three days after application. The population level of $B$. amyloliquefaciens CPA-8 remained stable during 45 days of cold storage at $0{ }^{\circ} \mathrm{C}$. The antagonistic strain was also monitored on non-target weeds under the tree canopy and on neighbouring untreated trees. On weeds in the treated plots up to $1.8 \times 10^{6} \mathrm{CFU}$ per $\mathrm{cm}^{2}$ of leaf surface were measured directly after application. Populations of the antagonist dropped during time in a similar pattern as found on treated leaves. Due to drift low amounts were also found on neighbouring trees which also dropped during time. No information is given on the occurrence of $B$. amyloliquefaciens and the specific strain B. amyloliquefaciens CPA-8 strain before application in the orchard to estimate possible background populations sizes.

\section{Lysobacter capsici}

The genus Lysobacter is of increasing interest as a source for new MBCAs. Among various species and strains of interest, L. capsici AZ78 has been studied more thoroughly. Its establishment and persistence in grape phyllosphere was studied by Segarra et al. (2015) using strain-specific qPCR. After application, population densities between $10^{5}$ and $10^{6}$ cells per gram leaf were measured. The population dropped by more than 100 times in one week, mainly due to weather conditions. Formulation of the cells increased their survival, but also the populations of formulated cells dropped by $90 \%$ during one week.

\section{Synthesis: system dynamics, population densities and risks}

The aim of this paper is to present a broader view on the relevance of environmental persistence in the context of risk assessment and approval of MBCAs. Several others have also questioned the necessity of costly and time-consuming data requirements for environmental fate of MBCAs (Alabouvette and Cordier 2011). This question of necessity is especially relevant since the need for MBCAs is greater than ever because the European Union 'promotes alternative approaches or techniques such as non-chemical alternatives to pesticides' in the framework of integrated pest and disease control as laid down in Directive 2009/128/EC (Anonymous 2009b). Nonetheless, appropriate safety criteria should be applied to any control measures, whether chemical or biological (Cook et al. 1996).

Therefore, this paper focuses on the many reasons why living organisms should be evaluated differently from chemical pesticides. Actually, the EU guidelines leaves space for a proportional interpretation of the data requirements on environmental risks as information is only needed "when relevant". This should be interpreted as "when ecologically relevant" or "relevant when it relates directly to food safety or human health in particular".

The basic characteristic of MBCAs is that they are naturally occurring living organism that are numerically enhanced to reduce populations of specific plant pathogens or pests. However, certain MBCAs may have a broader spectrum with a potential risk of side effects against non-target organisms related to the targeted organisms, e.g. the generalist entomopathogenic fungus Metarhizium brunneum can influence the behaviour of a predator of aphids (de Azevedo et al. 2018). MBCAs are typically isolated from environments similar to environments where they are applied. In biocontrol applications, their density is strongly increased by adding high amounts of the effective species or one of its strains. In contrast to chemicals, direct toxicity is not the main mechanism for control of harmful organisms, but rather a variety of mechanisms is involved, including competition, parasitism and activity of secondary metabolites (Köhl et al. 2019). Also, in contrast to chemicals, effects and residuals cannot be described as a breakdown of substances but rely on absorption and decline of populations due to a natural biological buffering of the environment. The natural decline of the population densities of the biocontrol agents limits the impact on the target organism and other organisms. This decline is due to competition for space and nutrients, as well as abiotic stress. Ecological theory predicts that in most cases any organism that is artificially enhanced to higher densities will naturally decline to levels within 
its natural density range for the location and its ecological characteristics (Ghoul and Mitri 2016).

It is crucial to recognize that fluctuations and shifts in the numbers of organisms are normal in any ecosystem, and the biological resilience of ecosystems buffers and limits the excessive increase of single organisms such as MBCAs. This also explains why it is hard to find and develop biocontrol agents that maintain at levels long enough to be effective. The literature screening and evaluation of a number of publications on the persistence of MBCAs, as was done in this paper, indicates that information is still scattered. More extensive data are available only for the MBCAs that are near market or already registered and used in the field. In all documented cases levels of MBCAs can be temporarily increased but always fall down to levels that are within normal fluctuations and ranges of microorganisms without strongly affecting microbial communities.

In this sense, biocontrol differs strongly from broad spectrum chemical pesticides because the former tend to more selectively suppressing the target organisms. In particular, the more broad spectrum chemical pesticides affect many more organisms at the same time and are likely to have broader effects on trophic interactions and functioning of the ecosystem. Whereas the persistence of a MBCA is limited by multiple interactions, the decline of chemicals is mediated by chemical breakdown of the toxic substances which are not naturally present in the ecosystem.

As for all living organisms, characteristics of MBCAs are determined by their genetics. Such genetic characteristics are not fixed for ever but subjected to mutation and selection processes so that the characteristics of a population change during time and can adapt to changing situations. Since genetic flexibility is an inherent part of life and a guarantee for a given species to adapt to a changing environment, also MBCAs are subjected to such a genetic flexibility. This may rise a fear that MBCAs may change their characteristics which may result in risks not considered in the regulations for approval as plant protection agents. Theoretically such a risk cannot be excluded for any species present in a given habitat. Application of a single MBCA adds only negligible amount of extra living cells of a naturally occurring microorganism to resident microbiota. Thus, MBCA applications do not add relevant additional risks for the highly unlikely adaptation and development of 'superior' species or strains in the environment. To our knowledge, such cases have not been reported.

A potential effect of resident populations of applied MBCA on harvested products may be their interference with other microorganisms that are important in food or feed processing. Fermentation processes during winemaking or production of silage may be affected because the compositions of naturally occurring or applied yeasts and bacteria important for fermentation processes may be disturbed by applied antagonists. Such possible side effects of resident populations of applied antagonists are considered during the development of MBCAs, e.g. for antagonist applications in grapevine (Escribano-Viana et al. 2018).

The concept of natural background levels as a reference to which densities should decline is of little value, in particular when endpoints cannot be defined from ecological theory or risk criteria. The precaution idea that nothing should change or that systems should return to natural equilibriums has no ecological base as it denies that in highly dynamic agro ecosystems perturbations, shifts and fluctuations are rules rather than exceptions. So far, and again in contrast to chemical pesticides, no side effects have been shown that go beyond the spatial boundaries of the agrosystem itself.

The maintenance of higher densities of MBCAs for at least part or even the whole growing season is an important property in making them effective. The control effect is often relatively slow and more temporal, sometimes requiring multiple treatments. This is quite different from the immediate control and quick breakdown requirements of chemical plant protection products. Therefore, a paradigm shift might be necessary for persistence criteria in the registration procedure, at least when the safety requirements for other properties of the MBCAs are fulfilled. In a tiered stepwise risk analysis persistence may only be relevant as a last step when other criteria give rise to potential risks associated with persistence.

In conclusion we state that data requirements for persistence could be more freely interpreted in cases where there is no a priori reason to assume that organisms will not be buffered by the agroecosystem where it naturally occurs at lower levels. More strict interpretations and requirements may need to be 
applied when the MBCA is not naturally occurring, behaves as a pathogen or is genetically modified.

Acknowledgements This study was funded by the Dutch Ministry of Agriculture, Nature and Food Quality (Programme Durable Plant Production, TKI-project 1406-130) with contributions from Artemis, 's-Gravenzande, the Netherlands, the International Biocontrol Manufacturers' Association (IBMA), Brussels, Belgium, and Linge Agroconsultancy b.v., Oosterhout, The Netherlands. We thank the members of the greenTEAM of the Board for the Authorisation of Plant Protection Products and Biocides (CTGB), Ede, The Netherlands, for their valuable comments on earlier versions of the manuscript.

\section{Compliance with ethical standards}

Conflict of interest The authors declare that they have no conflicts of interest.

Open Access This article is distributed under the terms of the Creative Commons Attribution 4.0 International License (http:// creativecommons.org/licenses/by/4.0/), which permits unrestricted use, distribution, and reproduction in any medium, provided you give appropriate credit to the original author(s) and the source, provide a link to the Creative Commons license, and indicate if changes were made.

\section{References}

Alabouvette C, Cordier C (2011) Risks of microbial biocontrol agents and regulation: are they in balance? In: Ehlers RU (ed) Regulation of biological control agents. Springer, Dordrecht, pp 157-173

Anonymous (2001) Commission Directive 2001/36/EC of 16 May 2001 amending Council Directive 91/414/EEC concerning the placing of plant protection products on the market. Off J Eur Commun L 164:1-38

Anonymous (2005) Council Directive 2005/25/EC of 14 March 2005 amending Annex VI to Directive 91/414/EEC as regards plant protection products containing micro-organisms. Off J Eur Commun L 90:1-34

Anonymous (2009a) Regulation (EC) No 1107/2009 of the European Parliament and of the Council of 21 October 2009 concerning the placing of plant protection products on the market and repealing Council Directives 79/117/ EEC and 91/414/EEC. Off J Eur Union L 309:1-50

Anonymous (2009b) Directive 2009/128/EC of the European Parliament and of the Council of 21 October 2009 establishing a framework for community action to achieve the sustainable use of pesticides. Off J Eur Union L 309:71-86

Anonymous (2011) Commission Regulation (EU) No 546/2011 of 10 June 2011 implementing Regulation (EC) No $1107 / 2009$ of the European Parliament and of the Council as regards uniform principles for evaluation and authorisation of plant protection products. Off J Eur Union L 155:127-175
Anonymous (2013a) Commission Regulation (EU) No 283/2013 of 1 March 2013 setting out the data requirements for active substances, in accordance with Regulation (EC) No 1107/2009 of the European Parliament and of the Council concerning the placing of plant protection products on the market. Off J Eur Union L 93:1-84

Anonymous (2013b) Commission Regulation (EU) No 284/2013 of 1 March 2013 setting out the data requirements for plant protection products, in accordance with Regulation (EC) No 1107/2009 of the European Parliament and of the Council concerning the placing of plant protection products on the market. Off $\mathrm{J}$ Eur Union L 93:85-152

Balog A, Hartel T, Loxdale HD, Wilson K (2017) Differences in the progress of the biopesticide revolution between the EU and other major crop-growing regions. Pest Manag Sci 73:2203-2208

Barberán A, Bates ST, Casamayor EO, Fierer N (2012) Using network analysis to explore co-occurrence patterns in soil microbial communities. ISME J 6:343-351

Bennett AJ, Leifert C, Whipps JM (2006) Survival of Coniothyrium minitans associated with sclerotia of Sclerotinia sclerotiorum in soil. Soil Biol Biochem 38:164-172

Bernard E, Larkin RP, Tavantzis S, Erich MS, Alyokhin A, Sewell G, Lannan A, Gross SD (2012) Compost, rapeseed rotation, and biocontrol agents significantly impact soil microbial communities in organic and conventional potato production systems. Appl Soil Ecol 52:29-41

Blagodatskaya E, Kuzyakov Y (2013) Active microorganisms in soil: critical review of estimation criteria and approaches. Soil Biol Biochem 67:192-211

Bonaterra A, Badosa E, Cabrefiga J, Francés J, Montesinos E (2012) Prospects and limitations of microbial pesticides for control of bacterial and fungal pomefruit tree diseases. Trees 26:215-226

Braun-Kiewnick A, Lehmann A, Smits THM, Duffy B, Dreo T (2011) Environmental monitoring of Pantoea agglomerans biocontrol strain E325 in Swiss orchards. Acta Hortic 896:425-430

Cook J, Bruckart WL, Coulson JR, Goettel MS, Humber RA, Lumsden RD, Maddox JV, McManus ML, Moore L, Meyer SF, Quimby PC, Stack JP, Vaughn JL (1996) Safety of microorganisms intended for pest and plant disease control: a framework for scientific evaluation. Biol Control $7: 333-351$

Cordier C, Edel-Hermann V, Martin-Laurent F, Blal B, Steinberg C, Alabouvette C (2007) SCAR-based real time PCR to identify a biocontrol strain (T1) of Trichoderma atroviride and study its population dynamics in soils. J Microbiol Methods 68:60-68

Daranas N, Bonaterra A, Francés J, Cabrefiga J, Montesinos E, Badosa E (2018) Monitoring viable cells of the biological control agent Lactobacillus plantarum PM411 in aerial plant surfaces by means of a strain-specific viability quantitative PCR method. Appl Environ Microb 84:e00107-e00118

de Azevedo AGC, Stuart RM, Sigsgaard L (2018) Presence of a generalist entomopathogenic fungus influences the oviposition behaviour of an aphid-specific predator. BioControl 63:655-664 
Deising HB, Gase I, Kubo Y (2017) The unpredictable risk imposed by microbial secondary metabolites: how safe is biological control of plant diseases? J Plant Dis Protect 124:413-419

Edel-Hermann V, Aimé S, Cordier C, Olivain C, Steinberg C, Alabouvette C (2011) Development of a strain-specific real-time PCR assay for the detection and quantification of the biological control agent Fo47 in root tissues. FEMS Microbiol Lett 322:34-40

Escribano-Viana R, Portu J, Garijo P, Gutiérrez AR, Santamaría P, López-Alfaro I, López R, González-Arenzana L (2018) Evaluating a preventive biological control agent applied on grapevines against Botrytis cinerea and its influence on winemaking. J Sci Food Agric 98:4517-4526

Faust K, Raes J (2012) Microbial interactions: from networks to models. Nat Rev Microbiol 10:538-550

Feng XM, Holmberg AIJ, Sundh I, Ricard T, Melin P (2011) Specific SCAR markers and multiplex real-time PCR for quantification of two Trichoderma biocontrol strains in environmental samples. BioControl 56:903-913

Frederiks C, Wesseler JHH (2018) A comparison of the EU and US regulatory frameworks for the active substance registration of microbial biological control agents. Pest Manag Sci 75:87-103

Ghoul M, Mitri S (2016) The ecology and evolution of microbial competition. Trends Microbiol 24:833-845

Glandorf DCM, Verheggen P, Jansen T, Jorritsma J-W, Smit E, Leeflang P, Wernars K, Thomashow LS, Laureijs E, Thomas-Oates JE, Bakker PAHM, van Loon LC (2001) Effect of genetically modified Pseudomonas putida WCS358r on the fungal rhizosphere microflora of field-grown wheat. Appl Environ Microbiol 67:3371-3378

Gotor-Vila A, Teixidó N, Usall J, Dashevskaya S, Torres R (2016) Development of a SCAR marker and a strainspecific genomic marker for the detection of the biocontrol agent strain CPA-8 Bacillus amyloliquefaciens (formerly B. subtilis). Ann Appl Biol 169:248-256

Holmberg AIJ, Melin P, Levenfors JP, Sundh I (2012) Fate and behaviour of a seed-applied Pseudomonas brassicacearum strain in a winter wheat field trial, as determined by analysis with SCAR markers. Biocontrol Sci Technol 22:379-392

Kapongo JP, Shipp L, Kevan P, Sutton JC (2008) Co-vectoring of Beauveria bassiana and Clonostachys rosea by bumble bees (Bombus impatiens) for control of insect pests and suppression of grey mould in greenhouse tomato and sweet pepper. Biol Control 46:508-514

Kiewnick S (2007) Practicalities of developing and registering microbial biological control agents. CAB Reviews: Perspectives in Agriculture, Veterinary Science, Nutrition and Natural Resources, vol 2, no. 013

Koch E, Becker JO, Berg G, Hauschild R, Jehle J, Köhl J, Smalla $\mathrm{K}$ (2018) Biocontrol of plant diseases is not an unsafe technology! J Plant Dis Protect 125:121-125

Köhl J, Postma J, Nicot P, Ruocco M (2011) Stepwise screening of microorganisms for commercial use in biological control of plant pathogenic fungi and bacteria. Biol Control 57:1-12

Köhl J, Kolnaar R, Ravensberg WJ (2019) Mode of action of microbial biological control agents against plant diseases: relevance beyond efficacy. Front Plant Sci 10:845
Kröber M, Wibberg D, Grosch R, Eikmeyer F, Verwaaijen B, Chowdhury SP, Hartmann A, Pühler A, Schlüter A (2014) Effect of the strain Bacillus amyloliquefaciens FZB42 on the microbial community in the rhizosphere of lettuce under field conditions analyzed by whole metagenome sequencing. Front Microbiol 5:252

Lilley AK, Hails RS, Cory JS, Bailey MJ (1997) The dispersal and establishment of pseudomonad populations in the phyllosphere of sugar beet by phytophagous caterpillars. FEMS Microbiol Ecol 24:151-157

Longa CMO, Savazzini F, Tosi S, Elad Y, Pertot I (2009) Evaluating the survival and environmental fate of the biocontrol agent Trichoderma atroviride $\mathrm{SC} 1$ in vineyards in northern Italy. J Appl Microbiol 106:1549-1557

Lugtenberg B (2018) Putting concerns for caution into perspective: microbial plant protection products are safe to use in agriculture. J Plant Dis Prot 125:127-129

Lugtenberg B, Rozen DE, Kamilova F (2017) Wars between microbes on roots and fruits. F1000Res 6:343

Mayerhofer J, Enkerli J, Zelger R, Strasser H (2015) Biological control of the European cockchafer: persistence of Beauveria brongniartii after long-term applications in the Euroregion Tyrol. BioControl 60:617-629

Mayerhofer J, Eckard S, Hartmann M, Grabenweger G, Widmer F, Leuchtmann A, Enkerli J (2017) Assessing effects of the entomopathogenic fungus Metarhizium brunneum on soil microbial communities in Agriotes spp. biological pest control. FEMS Microbiol Ecol 93:117

Mayerhofer J, Rauch H, Hartmann M, Widmer F, Gschwend F, Strasser H, Leuchtmann A, Enkerli J (2019) Response of soil microbial communities to the application of a formulated Metarhizium brunneum biocontrol strain. Biocontrol Sci Technol 29:547-564

Meyling NV, Pell JK, Eilenberg J (2006) Dispersal of Beauveria bassiana by the activity of nettle insects. J Invertebr Pathol 93:121-126

Mudgal S, De Toni A, Tostivint C, Hokkanen H, Chandler D (2013) Scientific support, literature review and data collection and analysis for risk assessment on microbial organisms used as active substance in plant protection products-lot 1 Environmental risk characterisation. EFSA Support Publ 10(12):518E. https://doi.org/10.2903/ sp.efsa.2013.EN-518

Natsch A, Keel C, Troxler J, Zala M, Von Albertini N, Defago G (1996) Importance of preferential flow and soil management in vertical transport of a biocontrol strain of Pseudomonas fluorescens in structured field soil. Appl Environ Microbiol 62:33-40

Ngosong C, Jarosch M, Raupp J, Neumann E, Ruess L (2010) The impact of farming practice on soil microorganisms and arbuscular mycorrhizal fungi: crop type versus long-term mineral and organic fertilization. Appl Soil Ecol 46:134-142

OECD (2018) Working group document on the risk assessment of secondary metabolites of microbial biocontrol agents. Series on Pesticides No. 98, ENV/JM/MONO(2018)33

Paulitz TC (2000) Population dynamics of biocontrol agents and pathogens in soils and rhizospheres. Eur J Plant Pathol 106:401-413

Perazzolli M, Antonielli L, Storari M, Puopolo G, Pancher M, Giovannini O, Pindo M, Pertot I (2014) Resilience of the 
natural phyllosphere microbiota of the grapevine to chemical and biological pesticides. Appl Environ Microbiol 80:3585-3596

Pujol M, Badosa E, Cabrefiga J, Montesinos E (2005) Development of a strain-specific quantitative method for monitoring Pseudomonas fluorescens EPS62e, a novel biocontrol agent of fire blight. FEMS Microbiol Lett 249:343-352

Raaijmakers JM, Mazzola M (2012) Diversity and natural functions of antibiotics produced by beneficial and plant pathogenic bacteria. Annu Rev Phytopathol 50:403-424

Ríos-Moreno A, Garrido-Jurado I, Resquín-Romero G, ArroyoManzanares N, Arce L, Quesada-Moraga E (2016) Destruxin A production by Metarhizium brunneum strains during transient endophytic colonisation of Solanum tuberosum. Biocontrol Sci Technol 26:1574-1585

Sadyś M, Adams-Groom B, Herbert RJ, Kennedy R (2016) Comparisons of fungal spore distributions using air sampling at Worcester, England (2006-2010). Aerobiologia 32:619-634

Sanzani SM, Li Destri Nicosia MG, Faedda R, Cacciola SO, Schena L (2014) Use of quantitative PCR detection methods to study biocontrol agents and phytopathogenic fungi and oomycetes in environmental samples. J Phytopathol 162:1-13

Scheepmaker JWA, Butt TM (2010) Natural and released inoculum levels of entomopathogenic fungal biocontrol agents in soil in relation to risk assessment and in accordance with EU regulations. Biocontrol Sci Technol 20:503-552

Scheepmaker JWA, van de Kassteele J (2011) Effects of chemical control agents and microbial biocontrol agents on numbers of non-target microbial soil organisms: a metaanalysis. Biocontrol Sci Techn 21:1225-1242

Segarra G, Puopolo G, Porcel-Rodríguez E, Giovannini O, Pertot I (2015) Monitoring Lysobacter capsici AZ78 using strain specific qPCR reveals the importance of the formulation for its survival in vineyards. FEMS Microbiol 363:fnv243

Soto-Muñoz L, Torres R, Usall J, Vinas I, Dashevskaya S, Teixido N (2015) Environmental monitoring of the biocontrol agent Pantoea agglomerans CPA-2 applied to citrus fruit at preharvest. Ann Appl Biol 167:250-261

Sundh I, Goettel MS (2013) Regulating biocontrol agents: a historical perspective and a critical examination comparing microbial and macrobial agents. BioControl 58:575-593

Thomas P, Sekhar AC (2016) Effects due to rhizospheric soil application of an antagonistic bacterial endophyte on native bacterial community and its survival in soil: a case study with Pseudomonas aeruginosa from banana. Front in Microbiol 7:493

Tilman D (1999) The ecological consequences of changes in biodiversity: a search for general principles. Ecology 80:1455-1474

Troxler J, Svercel M, Natsch A, Zala M, Keel C, MoenneLoccoz Y, Defago G (2012) Persistence of a biocontrol Pseudomonas inoculant as high populations of culturable and non-culturable cells in 200-cm-deep soil profiles. Soil Biol Biochem 44:122-129 van Elsas JD, Boersma FGH (2011) A review of molecular methods to study the microbiota of soil and the mycosphere. Eur J Soil Biol 47:77-87

van Lenteren JC, Bale J, Bigler F, Hokkanen HMT, Loomans AJM (2006) Assessing risks of releasing exotic biological control agents of arthropod pests. Annu Rev Entomol 51:609-634

van Lenteren JC, Bolckmans K, Köhl J, Ravensberg W, Urbaneja A (2017) Biological control using invertebrates and microorganisms: plenty of new opportunities. BioControl 63:39-59

van Veen JA, van Overbeek LS, van Elsas JD (1997) Fate and activity of micro-organisms introduced into soil. Microbiol Mol Biol Rev 61:121-135

Vazquez G, Melgarejo P, De Cal A, Larena I (2013) Persistence, survival, vertical dispersion, and horizontal spread of the biocontrol agent, Penicillium oxalicum strain 212, in different soil types. Appl Soil Ecol 67:27-36

Vilanova L, Teixidó N, Usall J, Balsells-Llauradó M, GotorVila A, Torres R (2018) Environmental fate and behaviour of the biocontrol agent Bacillus amyloliquefaciens CPA-8 after preharvest application to stone fruit. Pest Manag Sci 74:375-383

Weaver M, Vedenyapina E, Kenerley CM (2005) Fitness, persistence, and responsiveness of a genetically engineered strain of Trichoderma virens in soil mesocosms. Appl Soil Ecol 29:125-134

Wilson M, Lindow SE (1993) Release of recombinant microorganisms. Annu Rev Microbiol 47:913-944

Yang L, Li GQ, Long YQ, Hong GP, Jiang DH, Huang HC (2010) Effects of soil temperature and moisture on survival of Coniothyrium minitans conidia in central China. Biol Control 55:27-33

Yang T, Wei Z, Friman V-P, Xu Y, Shen Q, Kowalchuk GA, Jousset A (2017) Resource availability modulates biodiversity-invasion relationships by altering competitive interactions. Environ Microbiol 19:2984-2991

Yousef M, Garrido-Jurado I, Ruíz-Torres M, Quesada-Moraga E (2017) Reduction of adult olive fruit fly populations by targeting preimaginals in the soil with the entomopathogenic fungus Metarhizium brunneum. J Pest Sci 90:345-354

Yousef M, Alba-Ramírez C, Garrido Jurado I, Mateu J, Raya Díaz S, Valverde-García P, Quesada-Moraga E (2018) Metarhizium brunneum (Ascomycota; Hypocreales) treatments targeting olive fly in the soil for sustainable crop production. Front Plant Sci 9:1

Jürgen Köhl is studying biological control of plant diseases and is involved in selection programs for new MBCAs.

Kees Booij is a retired ecologist with wider expertise in agrobiodiversity, population dynamics of pests, modelling, food safety and emerging risks.

Rogier Kolnaar is a senior consultant in regulatory affairs. He coordinates regulatory projects of chemical and microbial plant protection products and their active substances. 
Willem Ravensberg has been developing MBCAs including dossiers for approval in the EU, USA and other countries. His activities include regulatory and governmental affairs related to biopesticides in industry associations and with authorities. 